NASA Technical Memorandum $89891^{\circ}$

\title{
Finite Element Implementation of Robinson's Unified Viscoplastic Model and Its Application to Some Uniaxial and Multiaxial Problems
}

V.K. Arya and A. Kaufman

Lewis Research Center

Cleveland, Ohio

June 1987

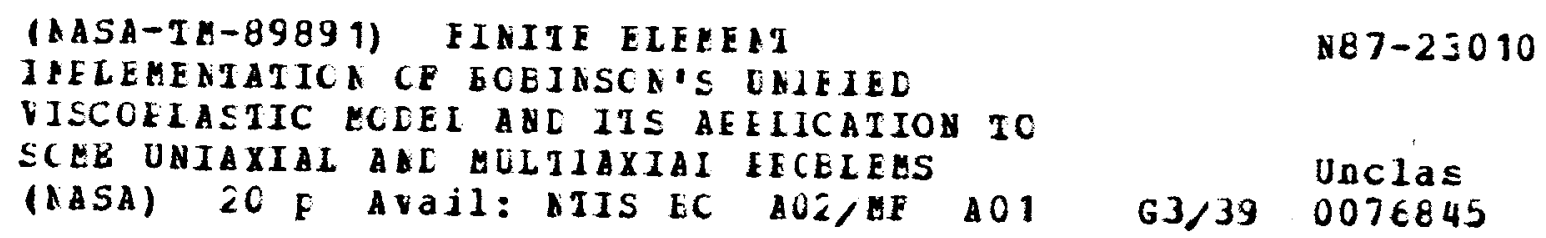


FINITE ELEMENT IMPLEMENTATION OF ROBINSON'S UNIFIED VISCOPLASTIC MODEL AND

ITS APPLICATION TO SOME UNIAXIAL AND MULTIAXIAL PROBLEMS

\author{
V.K. Arya* and A. Kaufmant \\ National Aeronautics and Space Administration \\ Lewis Research Center \\ Cleveland, Ohio 44135
}

SUMMARY

A description of the finite element implementation of Robinson's unified viscoplastic model into the General Purpose Finite Element Program - MARC, is presented. To demonstrate its application, the implementation is applied to some uniaxial and multiaxial problems. A comparison of the results for the multiaxial problem of a thick internally pressurized cylinder, obtained using the finite element implementation and an andytical solution, is also presented. Excellent agreement obtained confirms the correct finite element implementation of Robinson's model.

\title{
INTRODUCTION
}

Modern technological development in aerospace, nuclear, and many other industries has resulted in increasing use of materials at unprecedentediy higher temperatures. At high temperatures there is an increased propensity for inelastic deformation mechanisms of creep and plasticity. Realistic and rational designs of structural components operating at such elevated temperatures must rely on accurate constitutive model descriptions of these inelastic deformations in structural materials.

Conventional constitutive models which treat creep and plastic strains as separate noninteracting entities are incapable of dealing with observed creepplasticity interactions. The recent years have witnessed, therefore, a concerted effort by numerous researchers to develop constitutive models where the inelastic strain is not separated artificialiy into time-independent plastic and time-dependent creep components. These models - called the unified viscoplastic models - treat all inelastic strain (plasticity, creep, relaxation, etc.) as a unified and time dependent quantity and thus, automatically include any interactions. A good general overview of numerous viscoplastic models developed in recent years and their predictive capabilities is given by Walker (ref. 1) and Lindholm et a1. (ref. 2).

Unified viscoplastic models express the inelastic strain rates as a function of the current values of stress, temperature, and certain internal state variables. The models also include evolution (or growth) laws, whereby the rates of internal state variables are connected to the current values of these variables, stress, and temperature. The viscoplastic models proposed by different investigators employ different functional relations. Krieg (ref. 3) has, however, shown that most of these models possess the same basic skeletal

\footnotetext{
*National Research Council - NASA Research Associate.

tRetired.
} 
framework which generally includes two state variables - one scalar and the other tensorial - to account for isotropic and kinematic hardening, respectively.

In order to make these models more realistic, an attempt is made to incorporate as much material science as possible into the continuum model. As a result the mathematical structure of these models is, in general, very complex. Not only is the system of constitutive equations associated with these unified models highly nonlinear but also mathematically "stiff." Analytical tools commonly used for the solution of classical plasticity or creep problems are therefore rendered inapplicable for use with unified models. Many practical problems - such as those arising in the analysis of hot gas-path components of gas turbine engines and rocket engines for reusable space propulsion: systems - involve complex geometries as well as complex thermomechanical loading histories. For application of viscoplastic models to such intricate problems it is necessary that these models be implemented in General Purpose finite Element Codes such as MARC (ref. 4).

During the last several years the NASA Lewis Research Center has mounted an extensive in-house and external effort to implement some of the more commonly used viscoplastic models into the finite Element code - MARC. The viscoplastic models due to Walker (ref. 1) and Bodner (ref. 5) have already been implemented into the MARC and results of some nonlinear structural analyses using these implementations are avallable in references 1 and 6 . As a continued effort in this direction, the task of implementing Robinson's model (ref. 7) into MARC was undertaken. This report provides a brief description of this implementation and also presents the solutions of some illustrative problems.

\section{ROBINSON'S MODEL}

Robinson's model (ref. 7) is based on the concept of flow potential. The flow and growth laws for the internal variables are derived from this flow potential. The material behavior is elastic for all the stress states within the flow potential, and it is viscoplastic for all the stress states outside the flow potential. Assuming the displacements (and strains) to be small, the total strain rate, $\dot{\varepsilon}_{i j}$ is written as the sum of elastic, $\dot{\varepsilon}_{i j}$, and inelastic (including creep, plasticity, relaxation, etc.), $\dot{\varepsilon}\} \hat{j}$, components. In symbols

$$
\dot{\varepsilon}_{i j}=\dot{\varepsilon}_{i j}^{e l}+\dot{\varepsilon}_{i j}
$$

The material is assumed to be isotropic and Hooke's law is used to relate the elastic strain rate $\dot{\epsilon}_{i j}$ to the stress rate, $\dot{\sigma}_{i j} i . e$.

$$
\dot{\varepsilon}_{i j}^{e l}=\frac{1+v}{E} \dot{\sigma}_{i j}-\underline{v} \dot{\sigma}_{k k} \delta_{i j},
$$

where $E$ is the Young's modulus, $v$ is the Poisson's ratio, and $\delta_{i j}$ is the Kronecker delta. Following the Cartesian tensor notations, the repeated subscripts in equation (2) (and elsewhere) imply summation. 
The deviatoric stress, $S_{1 j}$, is defined as:

$$
S_{i j}=\sigma_{i j}-\frac{1}{3} \sigma_{k k} \delta_{i j} \text {. }
$$

The growth law governing the evolution of internal state variable, $\alpha_{i j}$, (which accounts for the kinematic hardening), is given by:

$$
\dot{\alpha}_{1 j}=h\left(\alpha_{k}\right) \dot{\varepsilon}_{i j}-r\left(\alpha_{k}\right) \alpha_{i j} .
$$

The form of this law is based on the well known Bailey-Orowan theory (ref. 8), which states that the high temperature deformation of materials takes place under the influence of two competing mechanisms denoted by the two terms in equation (4). The first term denotes the hardening process with accumulated deformation and the second term, a recovery or softening process proceeding with time. Under the steady-state conditions, these two mechanisms balance each other and consequently, $\dot{\alpha}_{1 j}=0$.

The flow law governing the inelastic strain rate, $\dot{\varepsilon}_{i j}$, is written as:

$$
2 \vec{\mu} \cdot \dot{\varepsilon}_{i j}^{\text {in }}=\left\{\begin{array}{l}
f(F) \sum_{1 j ;} F>0 \text { and } S_{i j} \sum_{i j}>0, \\
0 ; F \leq 0 \text { or } F>0 \text { and } S_{i j} \sum_{i j} \leq 0
\end{array}\right. \text {. }
$$

In equation (5), $\sum_{i j}$ denotes the effective stress, which is defined as:

$$
\Sigma_{i j}=S_{i j}-\alpha_{i j} .
$$

Particular forms for the functions $f(F), h\left(\alpha_{k}\right)$, and $r\left(\alpha_{k}\right)$ are taken as:

$$
\begin{gathered}
f(F)=\frac{F^{n}}{\sqrt{J_{2}}}, \\
h=\frac{2 \mu H}{G^{B}}, r=\frac{R G^{m-B}}{\sqrt{I_{2}}} ; G>G_{0} \text { and } S_{i j} \alpha_{i j}>0, \\
h=\frac{2 \mu H}{G_{0} B}, r=\frac{R G_{0}^{m-B}}{\sqrt{I_{2}}} ; G \leq G_{0} \text { or } S_{i j \alpha_{i j}} \leq 0,
\end{gathered}
$$


where

$$
\begin{aligned}
& \mathrm{J}_{2}=\frac{1}{2} \sum_{i j} \sum_{i j} ; \mathrm{F}=\frac{\mathrm{J}_{2}}{\kappa^{2}}-1 . \\
& \mathrm{I}_{2}=\frac{1}{2} \alpha_{i j} \alpha_{i j} ; \quad G=\frac{\sqrt{I_{2}}}{\kappa} .
\end{aligned}
$$

The parameters $\mu, H, n, m, B$, and $G_{0}$ are temperature independent constants. Effects of temperature are incorporated in the model through the temperature-dependent constants $\mu$ and $R$ using the following relations:

$$
\bar{\mu}=\mu \exp \left(-\theta_{1}\right) \text {, }
$$

and

$$
R=9.0 \times 10^{-8} \exp \left(\theta_{2}\right)
$$

In which

$$
\theta_{1}=(23.8 \theta-2635)\left(\frac{1}{811}-\frac{1}{\theta}\right) \text {, }
$$

and

$$
\theta_{2}=40000\left(\frac{1}{811}-\frac{1}{\theta}\right) .
$$

Here, $\theta$ denotes the absolute temperature in Kelvin $(K)$. The scalar state variable $\kappa$ in the above equations denotes the scalar threshold (Bingham) stress. Inelastic strain rate, $\dot{\varepsilon}_{i j}$, vanishes for all the values of second invariant $J_{2}$ below $\kappa$. The state variable $\kappa$ accounts for the isotropic hardening (or softening). Following Robinson (ref. 7), and for the material considered herein (2-1/4 $\mathrm{Cr}-1$ Mo steel), $\kappa$ has been taken as a temperature-independent constant. But, depending on the material, equations for the evolution of the isotropic state variable and for its dependence on temperature, may be necessary. An evolutionary equation for $\kappa$ may be found in reference 9 . The inequalities in equations (5) to (9) define boundaries across which the flow law and growth law change form discontinuously.

\section{DESCRIPTION OF MARC - PROGRAM}

Constitutive models are most advantageous if they can be used on a practical scale for the solution of problems faced by industry. The viscoplastic model developed by Robinson was therefore implemented into the General Purpose Finite Element Program - MARC (ref. 4). The MARC - program employs 
sophisticated integration algorithms and advanced finite element formulations, and is specially taflored to fit the requirements of a nonlinear structural analysis.

Robinson's model was implemented into MARC program by incorporating all the material nonlinearity into an initial load vector and treating it as a psuedo body force in finite element equilibrium equations. Since the constitutive equations associated with the viscoplastic model are, in general, "stiff," the subincrement technique is used to form the incremental constitutive equations corresponding to the finite load increment.

The subincrement technique essentially consists of splitting the finite load increment into a number of equal subincrements and integrating the constitutive equations of viscoplastic models over small subincrements to obtain an accurate representation of the incremental constitutive equations over the finite load increment. The explicit Euler forward difference method (with a self-adaptive integration strategy) was employed to integrate the constitutive equations over the subincrements. The technique is found to work efficientiy and accurately, even for large finite element load increments, provided the subincrements are small enough to preserve the stability of Euler forward method. However, it is difficult for the user to select efficient subincremental steps, and there is a considerable incentive to use as few subincrements as possible without affecting the stability of constitutive differential equations associated with the model.

To familiarize the reader with its operation, a brief introduction to MARC - Program is appropriate. This introduction, taken from references 1 and 10, is presented in the following paragraphs.

"The principal of virtual work may be used to generate the MARC nonlinear equilibrium equations governing the incremental response of the structure to an increment of load. In evaluating the nonlinear structural response of a component, the program assumes that the load history is divided into incrementally applied loads. Each load step is sequentially analyzed as a linear matrix problem using an appropriate stiffness matrix and load vector. Although each load step uses linear matrix methods to solve the incremental equations, the incremental equations themselves are nonlinear since the load vector will depend on the displacement increment obtained in the solution of incremental equilibrium equations.

The principle of virtual work may be written, for applied external point loads $P_{j}$, or displacement $u_{j}$, in the form:

$$
\sum \int_{V} \delta \varepsilon_{i}^{\top} \sigma_{i} d V=\delta u_{i}^{\top} P_{i}
$$

where the integral extends over the volume, $v$, of each finite element and the summation sign extends to all the elements in the structure.

In equation (15) the virtual displacement vector $\delta_{u i}$ is related to the virtual strain vector $\delta e_{1}$ through the relationship

$$
\delta \varepsilon_{i}=B_{i j} \delta_{u j} \quad \text { or } \quad \delta \varepsilon_{i}^{\top}=\delta_{u j}^{\top} B_{i j}^{\top} \text {, }
$$


where Bij is the strain displacement matrix and the superscript $T$ denotes Lransposilion. Since $\delta_{U 1}$ is an arbitrary virtual displacement vector. equations $(15)$ and $(16)$ may be written in the form:

$$
\sum \int_{V} B_{i j}^{T} \sigma_{j} d V=P_{i}
$$

This relation expresses the equilibrium of structure when the applied load vector is. $P_{j}$ and the stress vector is $\sigma_{j}$. If an incremental load $\Delta P_{j}$ is applied to the structure and the stress vector changes to $\sigma_{j}+\Delta \sigma_{j}$, the relation expressing the equilibrium of the structure at the end of the incremental load application may be written as:

$$
\sum \int_{V} B_{i j}^{T}\left(\sigma_{j}+\Delta \sigma_{j}\right) d V=P_{i}+\Delta P_{i}
$$

Hence, the relation expressing the equilibrium of the structure during the application of the incremental load vector $\Delta P_{1}$ is obtained from equations (17) and (18) by subtraction in the form:

$$
\sum \int_{V} B_{i j}^{\top} \Delta \sigma_{j} d V=\Delta P_{i}
$$

The MARC code allows the user to implement very general constitutive relationships into the program by means of the user subroutine HYPELA. Within this subroutine, the user must specify the values of the elasticity matrix $D_{i j}$ and the inelastic stress increment vector $\Delta \zeta j$ in the incremental vector constitutive relationship:

$$
\Delta \sigma_{i}=0_{i j}\left(\Delta \varepsilon_{j}-\delta_{j}^{\alpha} \Delta \theta\right)-\Delta \zeta_{i} .
$$

The inelastic stress increment vector $\Delta \zeta_{\uparrow}$ is computed in HYPELA using the constitutive relationships of the viscoplastic model.

In equation (20), $\alpha$ denotes the coefficient of thermal expansion and $\delta_{j}$ is the vector kronecker delta symbol,

$$
\delta_{j}=\left\{\begin{array}{lll}
1 & \text { if } & 0 \leq j \leq 3 \\
0 & \text { if } & 3<j \leq 6
\end{array} .\right.
$$

For the viscoplastic models, "the incremental inelastic stress vector $\Delta \zeta_{1}$ depends in a highly nonlinear manner on the incremental strain vector $\Delta \varepsilon f$. Since $\Delta \varepsilon \dot{q}=B_{i j} \Delta_{u j}$, the incremental inelastic stress vector $\Delta \zeta_{j}$ depends in a highly nonlinear manner on the nodal displacement vector $\Delta_{u j}$, so that $\Delta \zeta i=\Delta \zeta\left(\Delta_{u j}\right)$.

Substitution of equation (2) into equation (19) produces the incremental equilibrium equations for MARC in the form:

$$
\sum k_{i j} \Delta u_{j}=\Delta P_{i}+\Delta R_{i}+\sum \int_{V} B_{i j}^{T} \Delta \zeta_{j} d V+\sum \int_{V} B_{i j}^{\top} \delta_{j} \alpha \Delta \theta d V,
$$


where $k_{i j}$ is the elemental elastic stiffness matrix defined by the relation:

$$
K_{i j}=\int_{V} B_{i k}^{T} D_{k \ell}^{B}{ }^{B} j d V
$$

The vector $\Delta R_{1}$ is the residual load correction vector or out-of-equilibrium force vector from the preceding load increment:

$$
\Delta R_{i}=P_{i}-\sum \int_{V} B_{i j} \sigma_{j} d V
$$

which is added to the current increment in order to restore the structure to equilibrium. The nonlinearity in the incremental equilibrium relationship. defined in equation (22), arises because the inelastic stress increment vector $\Delta \zeta_{j}$ depends nonlinearly on the displacement increment vector $\Delta_{u j}$. Values of $D_{i j}$ and $\Delta \zeta_{j}$ appropriate to the current incremental load step are returned to the main program by subroutine HYPELA and the incremental equilibrium relations in equation (22) are solved by successive iterations.

The solution of the incremental equilibrium equations in (22) is accomplished within the MARC code by the following algorithm. At the start of the increment the user subroutine HYPELA is entered to determine the elasticity matrix $D_{i j}$ and the incremental inelastic stress vector $\Delta \zeta_{j}$. On entry to the subroutine, the input consists of the strain increment vector $\Delta \varepsilon$, the temperature increment $\Delta \theta$, the time increment $\Delta t$ over which the incremental external load vector $\Delta P_{j}$ is applied to the structure, and the values of stress, strain, temperature and viscoplastic state variables at the beginning of the increment. Since the incremental strain vector, $\Delta \varepsilon_{j}=B_{1 j} \Delta_{u j}$, can only be accurately determined after the solution to the incremental equilibrium relationship in equation (22) has yielded the correct incremental solution vector $\Delta_{u j}$, the strain increment vector $\Delta \varepsilon_{j}$ initially used to generate the inelastic stress increment vector $\Delta \zeta_{j}$ must be estimated. The initial estimate for $\Delta \varepsilon_{j}$ is assumed to be the value obtained for $\Delta \varepsilon_{j}$ in the preceding increment. On exit from subroutine HYPELA the elasticity matrix $D_{i j}$ and the estimated inelastic stress increment vector $\Delta \zeta_{j}$ are passed into the main program. After the values of $D_{i j}$ and $\Delta \zeta j$ are obtained for each integration point in the structure, the incremental equilibrium relationship in equation (22) is assembled and solved for the incremental node displacement vector $\Delta_{u j}$. The incremental strain vector, $\delta \varepsilon_{j}=B_{i j} \Delta_{u j}$, is then computed and compared with the initial guess for $\Delta \varepsilon_{j}$ used to generate the inelastic incremental stress vector $\Delta \zeta_{j}$. If this incremental strain vector is equal, within a user specified tolerance, to the incremental strain vector used to compute $\Delta \zeta_{j}$ in the assembly phase, the solution is assumed to have converged. otherwise, the updated strain increment vector, obtained from the solution of the equilibrium relations in equation (22), is passed into subroutine HYPELA, a new vector, $\Delta \zeta_{j}$, is computed and the equilibrium equations resolved to yield an improved value of $\Delta_{u} i$ and $\Delta \varepsilon_{i}$. The process is repeated until the value of vector $\Delta \varepsilon_{1}$ on the assembly phase is equal, within a user specified tolerance, to the value of the vector $\Delta \varepsilon_{j}$ on the solution phase. After convergence is achieved, the temperature, stress vector, strain vector and viscoplastic state variabies are updated by adding the incremental values generated during the current increment to the values of these variables at the beginning of the increment. The program then passes on to the next load increment where the process is repeated." 
The MARC - Program provides the user a convenient way of implementing and integrating the constitutive equations of Robinson's model through the user subroutine HYPELA. The details of subroutine HYPELA may be found in reference 4. The subroutine HYPELA written for Robinson's model and the selfadaptive integration scheme used in it, follow essentially the same structure as given in reference 10 for Walker's model.

The discontinuous boundaries in Robinson's model should be smoothed to facilitate numerical computations using the FEM. The smoothing is achieved by defining a spline function $P(x)$ on the interval $(-1,1)$ as:

$$
P(x)=\left\{\begin{array}{l}
\frac{(1+x)^{2}}{2} ;-1 \leq x<0 \\
1-\frac{(1-x)^{2}}{2} ; 0 \leq x \leq 1 \\
1 ; x>1 \\
0 ; x<-1 .
\end{array}\right.
$$

The function $F$ in equation (1) is then replaced by a function $\mathscr{F}$ defined as:

$$
\mathscr{F}=P\left(\frac{S_{i j} \sum_{i j}}{W_{1}}\right)\langle F\rangle \text {, }
$$

where the angular brackets denote:

$$
\langle x\rangle=\left\{\begin{array}{l}
x ; x \geq 0 \\
0 ; x<0,
\end{array},\right.
$$

and the weighting function $w_{1}$ is to be selected by the user. The use of

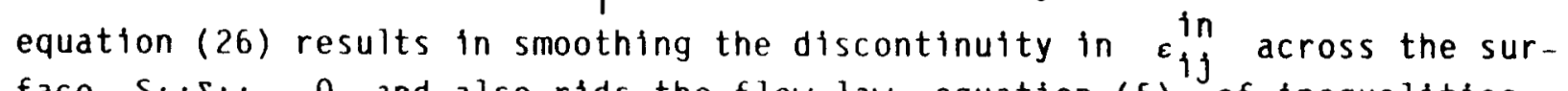
face, $S_{i j} \sum_{i j}=0$, and also rids the flow law, equation (5). of inequalities.

The discontinuties in aij are removed by first replacing the function $G$ by $G_{1}$, i.e.

$$
G_{1}=\left\{\begin{array}{l}
G ; G \geq 2 G_{0} \\
\frac{G^{2}}{4 G_{0}}+G_{0} ; G<2 G_{0},
\end{array}\right.
$$

and then defining

$$
G=\left(G_{1}-G_{0}\right) P\left(\frac{S_{i j}^{\alpha} i j}{W_{2}}\right)+G_{0} .
$$


lquations (8) and (9) may now be rewritten as:

$$
h=\frac{2 \mu H}{G^{B}} \text {, }
$$

and

$$
r=\frac{R G^{\mathrm{m}-\beta}}{\sqrt{\mathrm{I}_{2}}}
$$

With these values of $h$ and $r$, the growth law, equation (6), reduces to a single expression with a smooth transition across the surface, $S_{i j \alpha i j}=0$, in the space and now contains no inequalities. The weighting function $W_{2}$ is again again to be selected by the user. The computations and results presented in the following sections used:

$$
W_{1}=W_{2}=10^{-2}\left(k s j^{2}\right)=0.475\left(\mathrm{MPa}^{2}\right) .
$$

\section{NUMERICAL VALUES OF THE CONSTANTS}

The numerical values of the parameters appearing in equations (1) to (14) for the alloy 2-1/4 $\mathrm{Cr}-1$ Mo steel were taken from Robinson and Swindeman, (ref. 7). The values are:

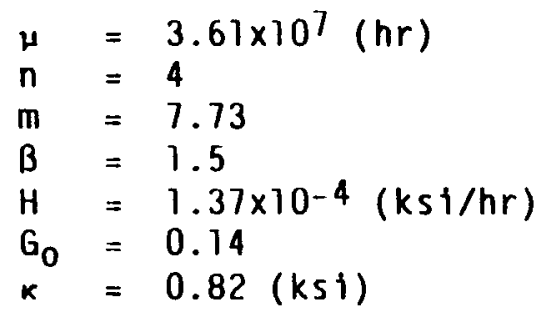

The values of Young's modulus, $E$, and the Poisson's ratio, $v$ are assumed to be temperature dependent. The following polynomial expressions in temperature taken from Sartory (ref. 11), are used to estimate the values of $E$ and $v$ :

$$
E=31100.0-13.59 T+0.2505 \times 10^{-5} T^{2}-0.2007 \times 10^{-13} T^{3} \text { (ksi), }
$$

and

$$
v=0.524+0.154 \times 10^{-3} \mathrm{~T}-0.126 \times 10^{-6} \mathrm{~T}^{3} .
$$

Here, $T$ denotes temperature in degree Farenheit. 


\section{APPLICATION TO PROBLEMS}

\section{Uniaxial Problems}

Some illustrative problems were solved to demonstrate the implementation of Robinson's model into MARC through the subroutine HYPELA. The values of constants listed in the preceding sections were utilized for these computations. The thermal and mechanical loadings used to generate the hysteresis loops using MARC are shown in figure 1.

Figures 2 through 4 show the hysteresis loops generated by MARC using Robinson's mode1. The loops shown in these figures are at strain rates of $0.0004,0.004$, and $0.04 / \mathrm{min}$, respectively. The strain range is \pm 0.2 percent approximately. The cyclic mechanical loading used in generating the hysteresis loops is shown in figure $1(\mathrm{a})$ and the thermal loading is constant at $800^{\circ} \mathrm{F}$. These figures exhibit the results for two and one-quarter of a cycle. Figure 5 summarizes the stablized hysteretic loops for the three strain rate coilditions.

The results for combined in-phase thermomechanical loading, shown in figure $7(b)$, are depicted in figures 6 to 8 . These figures show the hysteresis loops for the same strain-rates and strain range as mentioned above. Figure 9 summarizes the stable hysteretic loops for this type of thermomechanical loading.

As a further example, the combined out-phase thermal and mechanical loading shown in figure $1(\mathrm{c})$, was used to generate the hysteresis loops at strain rates of $0.0004,0.004$, and $0.04 / \mathrm{min}$, respectively. The strain range, as before, is \pm 0.2 percent. Figures 10 to 12 show the results of these calculations for two and a one-quarter of loading cycles. The stable hysteretic loops for the three strain rates are plotted in figure 13. The loops of figures 9 and 13 are identical if the signs of stresses and strains are simply reversed. Figures 2 to 13 depict the effects of different strain rates and different types of thermomechanical loadings.

Figure 14 compares the isothermal hysteretic loops (1000 $\left.{ }^{\circ} \mathrm{F}\right)$ generated by MARC code with those obtained experimentally by Robinson and Swindeman (ref. 7). The strain rates are 0.0004 and $0.04 / \mathrm{min}$, respectively and the strain range +0.32 percent, approximately. The excellent agreement between the theoretical and experimental loops verifies the correct finite element implementation of Robinson's model.

\section{Multiaxial Problem}

In order to have a further verification and application of the finite element implementation of Robinson's constitutive equations, the implementation was applied to a multiaxial problem. The problem selected was that of a thick-walled cylinder subjected to internal pressure under isothermal ( $800^{\circ} \mathrm{F}$ ) conditions. The inner and outer radif of the cylinder were taken to be $0.16 \mathrm{in.}(4.06 \mathrm{~mm})$ and $0.25 \mathrm{in} .(6.35 \mathrm{~mm})$ and the internal pressure as $3.65 \mathrm{ksi}$ $(25.17 \mathrm{MPa})$. The results of these computations have been plotted in figures 15 and 16 . 
Figure 15 shows the hoop (circumferential) stress distribution across the wall of cylinder using the Robinson's model and the FE Code - MARC. The curves plotted at different values of time show the redistribution of stress as predicted by Robinson's model. The hoop strain distribution obtained using Robinson's model and MARC has been plotted in figure 16 for different values of time.

In order to verify the results from MARC and to ensure the correct implementation of Robinson's model in the code, the analytical solution for the problem of a thick, internally pressurized cylinder was obtained. The following section presents briefly the details of the analytical solution.

\section{ANALYTICAL SOLUTION FOR THICK-CYLINDER}

In the following, the subscripts $r, \varphi$, and $z$ refer to the radial, hoop (circumferential) and axial directions respectively. Symbol, o denotes the stress and the symbol $c$, strain.

Following Saada (ref. 12), the equation of equilibrium may be written as:

$$
\frac{\partial \sigma_{r}}{\partial r}=\frac{\sigma_{\varphi}-\sigma_{r}}{r} .
$$

Assuming the total strain rate to be composed of elastic and inelastic components, the constitutive relations in the rate form may be written as:

$$
\begin{aligned}
& \dot{\varepsilon}_{r}=\frac{1}{E}\left[\dot{\sigma}_{r}-v\left(\dot{\sigma}_{\varphi}+\dot{\sigma}_{z}\right)\right]+\dot{\varepsilon}_{r}^{i n}, \\
& \dot{\varepsilon}_{\varphi}=\frac{1}{E}\left[\dot{\sigma}_{\varphi}-v\left(\dot{\sigma}_{z}+\dot{\sigma}_{r}\right)\right]+\dot{\varepsilon}_{\varphi}^{i n},
\end{aligned}
$$

and

$$
\dot{\varepsilon}_{z}=\frac{1}{E}\left[\dot{\sigma}_{z}-v\left(\dot{\sigma}_{r}+\dot{\sigma}_{\varphi}\right)\right]+\dot{\varepsilon}_{Z}^{\text {in }} .
$$

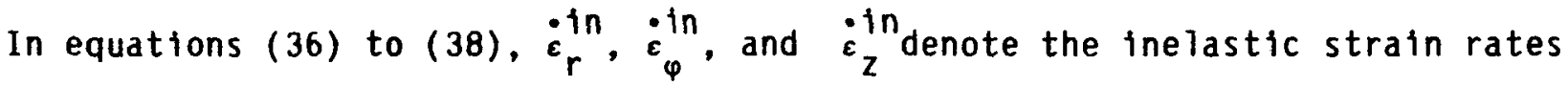
in the respective directions. These rates are obtained using the constitutive equations of Robinson's model. The dot over a symbol indicates differentiation with respect to time, $t$.

The strain-rate-compatibility relation is:

$$
\frac{\partial \dot{\varepsilon} \varphi}{\partial r}+\frac{\dot{\varepsilon}_{\varphi}-\dot{\varepsilon}_{r}}{r}=0 \text {. }
$$


and, the boundary conditions of the problem are:

$$
\begin{array}{ll}
\sigma_{r}=-p & \text { at the inner radius } r=a, \\
\sigma_{r}=0 & \text { at the outer radius } r=b .
\end{array}
$$

Using equations (35) to (40), and after some algebraic manipulations, one may obtain the following expression for the stress rates:

$$
\begin{aligned}
& \dot{\sigma}_{r}=\frac{E}{2\left(1-v^{2}\right)}\left(\int_{a}^{r} \frac{\dot{\varepsilon}_{r}^{i n}-\dot{\varepsilon}_{\varphi}^{i n}}{r} d r-\frac{\left(r^{2}-a^{2}\right)}{\left(b^{2}-a^{2}\right)} \cdot \frac{b^{2}}{r^{2}} \int_{a}^{b} \frac{\dot{\varepsilon}_{r}^{i n}-\dot{\varepsilon}_{\varphi}^{\text {in }}}{r} d r\right) \\
& +\frac{E(1-2 v)}{2\left(1-v^{2}\right)} \cdot \frac{1}{r^{2}}\left(\int_{a}^{r} r \dot{\varepsilon}_{z}^{i n} d r-\frac{\left(r^{2}-a^{2}\right)}{\left(b^{2}-a^{2}\right)} \int_{a}^{b} r \dot{\varepsilon}_{z}^{i n} d r\right) \text {, } \\
& \dot{\sigma}_{\varphi}=\frac{E}{2\left(1-v^{2}\right)}\left(\int_{a}^{r} \frac{\dot{\varepsilon}_{r}^{i n}-\dot{\varepsilon}_{\varphi}^{i n}}{r} d r-\frac{\left(r^{2}+a^{2}\right)}{\left(b^{2}-a^{2}\right)} \cdot \frac{b^{2}}{r^{2}} \int_{a}^{b} \frac{\dot{\varepsilon}_{r}^{i n}-\dot{\varepsilon}_{\varphi}^{i n}}{r} d r\right) \\
& -\frac{E(1-2 v)}{21-v^{2}} \cdot \frac{1}{r^{2}}\left(\int_{a}^{r} r \dot{\varepsilon}_{z}^{i n} d r+\frac{\left(r^{2}+a^{2}\right)}{\left(b^{2}-a^{2}\right)} \int_{a}^{b} r \dot{\varepsilon}_{z}^{i n} d r\right)-\frac{E}{\left(1-v^{2}\right)}\left(\dot{\varepsilon}_{\varphi}^{i n}+v \dot{\varepsilon}_{z}^{i n}\right) \text {, }
\end{aligned}
$$

and

$$
\begin{aligned}
& \dot{\sigma}_{z}=\frac{E v}{\left(1-v^{2}\right)}\left(\int_{a}^{r} \frac{\dot{\varepsilon}_{r}^{i n}-\dot{\varepsilon}_{\varphi}^{i n}}{r} d r-\frac{b^{2}}{\left(b^{2}-a^{2}\right)} \int_{a}^{b} \frac{\dot{\varepsilon}_{r}^{i n}-\dot{\varepsilon}_{\varphi}^{i n}}{r} d r\right) \\
& +\frac{E(2-v)}{\left(1-v^{2}\right)} \cdot \frac{1}{\left(b^{2}-a^{2}\right)} \int_{a}^{b} r_{z}^{i n} d r+\frac{E}{\left(1-v^{2}\right)}\left\{v \dot{\varepsilon}_{r}^{i n}-(1-v) \dot{\varepsilon}_{z}^{i n}\right\} .
\end{aligned}
$$

These equations together with the constitutive equations of Robinson's model were integrated using the explicit Euler forward method with an automatic time-step integration strategy proposed by Arya et a 1. (ref. 13). The integration yields the stresses and strains at different times and radic of the cylinder. The spatial integrals in equations (41) to (43) were evaluated using the Trapezoidal rule.

The values of the hoop (circumferential) stress across the wall of the cylinder at different times have been plotted in figure 17 . Figure 18 exhibits 
the values of hoop strain at different times in the cylinder obtained using the analytical solution. The values of stress and strain from the MARC program have also been plotted in these figures for comparison of results. The excellent agreement between the MARC and the analytical solutions verifies the correct implementation of Robinson's model into FE Code - MARC. The finite element implementation can therefore be safely employed to carry out the nonlinear finite element analysis of structures with more complex geometries such as gas turbine airfolls subjected to complex thermal and mechanical loadings in aerospace propulsion engines. The results of such analyses will be reported subsequentiy.

\section{SUMMARY AND CONCLUSIONS}

1. Robinson's unified viscoplastic model has been successfully implemented into the Finite Element Code - MARC.

2. Some uniaxial and multiaxial problems have been solved using the implementation. The results obtained for a multiaxial thick cylinder problem using the implementation compare very well with the corresponding results from the analytical solution and verify the correct finite element implementation of Robinson's model.

3. The implementation can now be used for the analysis of more complex problems, both in geometry and loadings, from aerospace and other industries, where analytical methods become inapplicable, or are too cumbersome to apply.

\section{REFERENCES}

1. Walker, K.P.: Research and Development Program for Nonlinear Structural Modeling with Advanced Time-Temperature Dependent Constitutive Relationships. (PWA-5700-50, Pratt and Whitney Aircraft; NASA Contract NAS3-22055) NASA CR-165533, 1981.

2. Lindholm, U.S., et al.: Constitutive Modeling for Isotropic Materials. NASA CR-174718, 1984.

3. Krieg, R.D.: Numerical Integration of Some New Unified Plasticity-Creep Formulations. Transactions of the 4 th International Conference on Structural Mechanics in Reactor Technology, Commission of the European Communities, Luxembourg, 1977, Paper No. M6/4.

4. MARC General Purpose Finite Element Program. MARC Analys is Research Corporation, Palo Alto, CA, 1983.

5. Bodner, S.R.; and Partom, Y.: Constitutive Equations for ElasticViscoplastic Strain Hardening Materials. J. Appl. Mech., vol. 42, no. 2, June 1975, pp. 385-389.

6. Kaufman, A., et al.: Structural Analysis of Turbine Blades Using Unified Constitutive Models. NASA TM-88807, 1986. 


\section{CREYMI. PAGE IS \\ OF POOR QUALTY}

7. Robinson, D.N.; and Swindeman, R.W.: Unified Creep-Plasticity Constitutive Equations for 2-1/4 Cr-1 Mo Steel at Elevated Temperature. ORNL/TM-8444, Oct. 1982.

8. Orowan, E.: The Creep of Metals. J. West Scotl Iron Steel Inst., vol. 54, 1946-47, pp. 45-96.

9. Robinson, D.N.; and Bartolotta, P.A.: Viscoplastic Constitutive Relationships with Dependence on Thermomechanical History. NASA CR-174836, 1985.

10. Cassenti, B.N.: Research and Development Program for the Development of Advanced Time-Temperature Dependent Constitutive Relationships.

(R83-956077-1, -2, United Technologies Research Center; NASA Contract NAS3-23273) NASA CR-168191, 1983.

11. Sartory, W.K.: Inelastic Ratchetting Analysis of the 2-1/4 Cr-1 Mo Steel to Type 316 Stainless Steel Dissimilar Metal Weldment Region of Specimen TTT-3. ORNL-5512, Mar. 1979.

12. Saada, A.S.: Elasticity Theory and Applications. Pergamon Press Inc., 1974 .

13. Arya, V.K.; Hornberger, K.; and Stamm, H.: On the Numerical Integration of Viscoplastic Models. KFK-4082, May 1986.

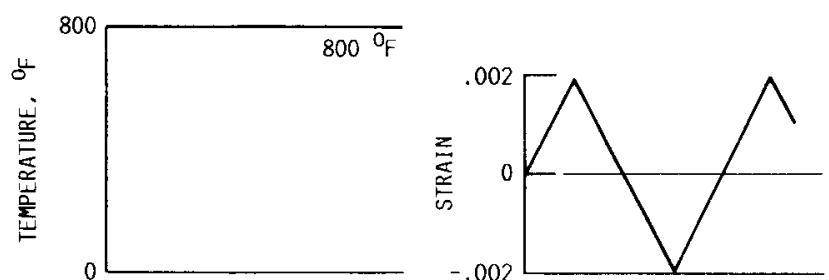

(A) ISOTHERMAL LOADING.
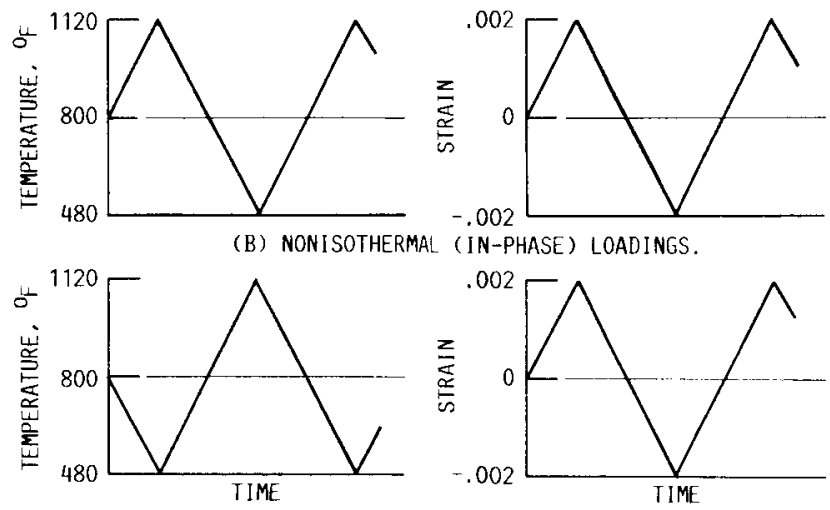

(C) NONISOTHERMAL (OUT-PHASE) LOADINGS.

FIGURE 1. - CYCLIC THERMAL AND MECHANICAL LOADINGS USEDD IO GENERATE HYSTERESIS LOOPS BY FE CODE-MARC.

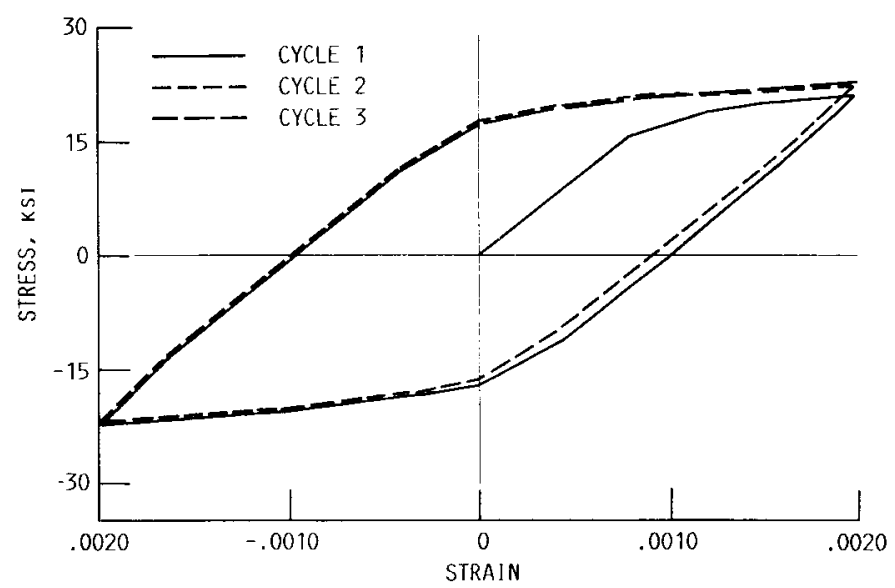

FIGURE 2. - HYSTERESIS LOOP USING ROBINSON'S MODEL AT $800^{\circ} \mathrm{F}$ (STRAIN RATE $=0.0004 / \mathrm{MIN})$. 


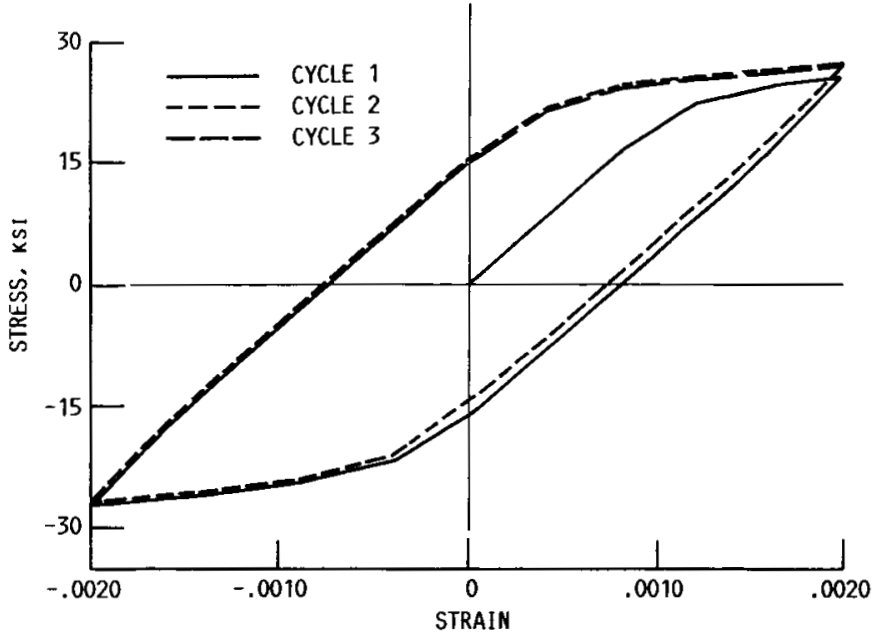

FIGURE 3. - HYSTERESIS LOOP USING ROBINSON'S MODEL AT $800^{\circ} \mathrm{F}$ (STRAIN RATE $=0.004 \mathrm{MIN})$.

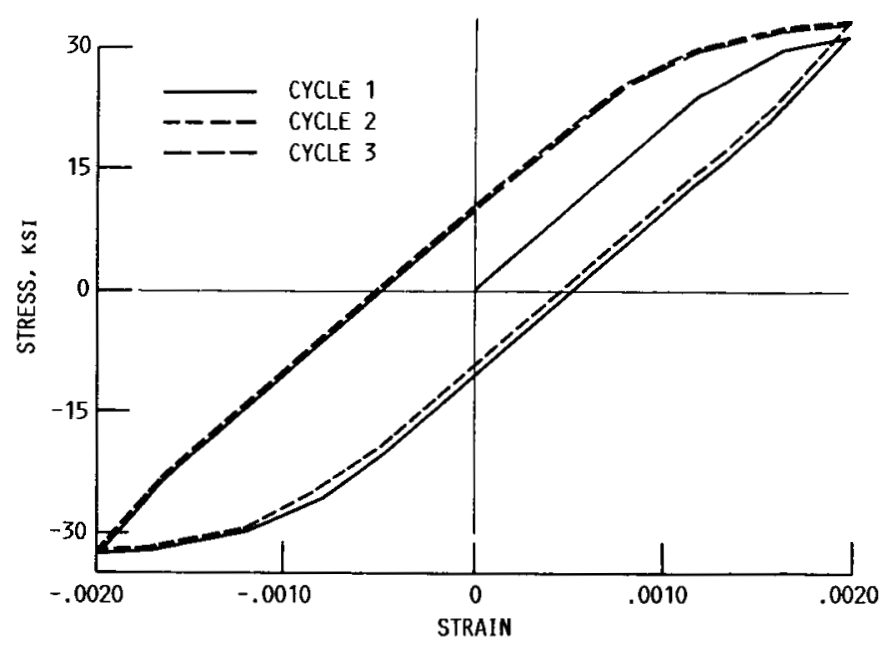

FIGURE 4. - HYSTERESIS LOOP USING ROBINSON'S MODEL AT $800^{\circ} \mathrm{F}$ (STRAIN RATE $=0.04 / \mathrm{MIN})$.

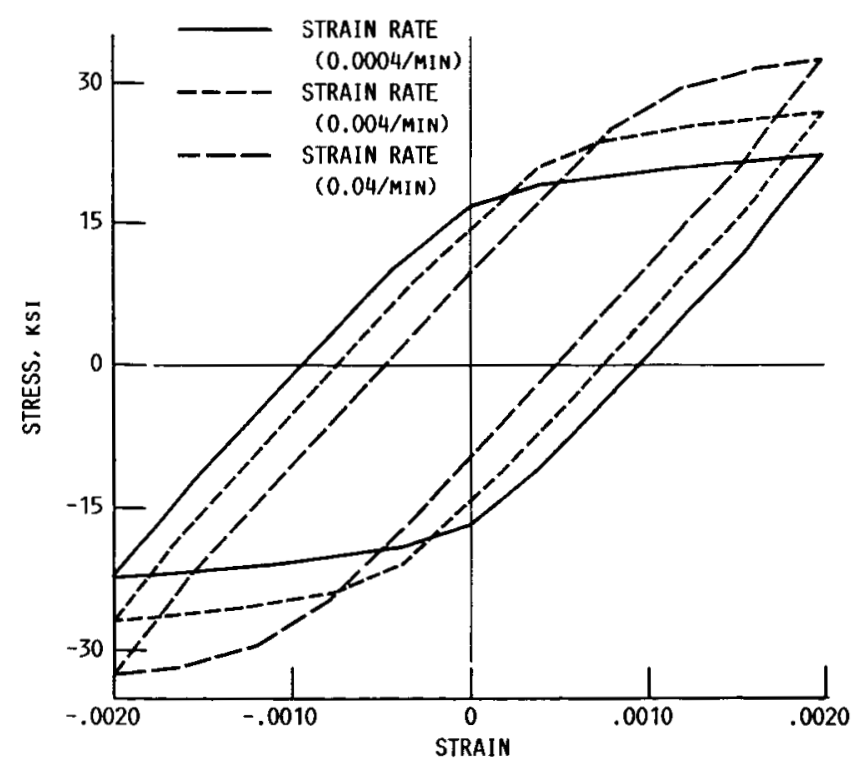

FIGURE 5. - HYSTERESIS LOOPS AT DIFFERENT STRAIN RATES USING ROBINSON'S MODEL-ISOTHERMAL CASE $\left(800^{\circ} \mathrm{F}\right)$.

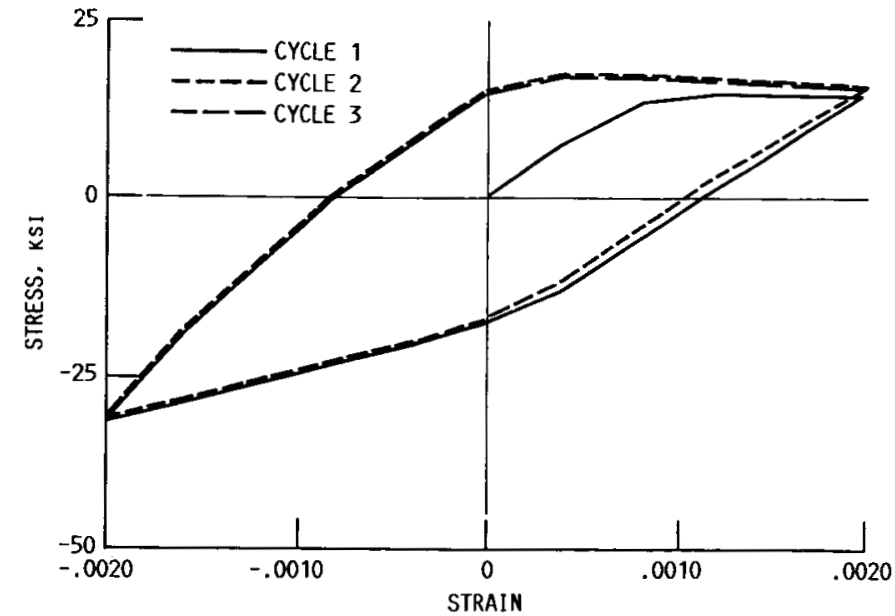

FIGURE 6. - HYSTERESIS LOOP USING ROBINSON'S MODEL-NONISOTHERMAL CASE (STRAIN RATE $=0.0004 / \mathrm{MIN})$, IN-PHASE THERMAL AND MECHANICAL LOADINGS. 


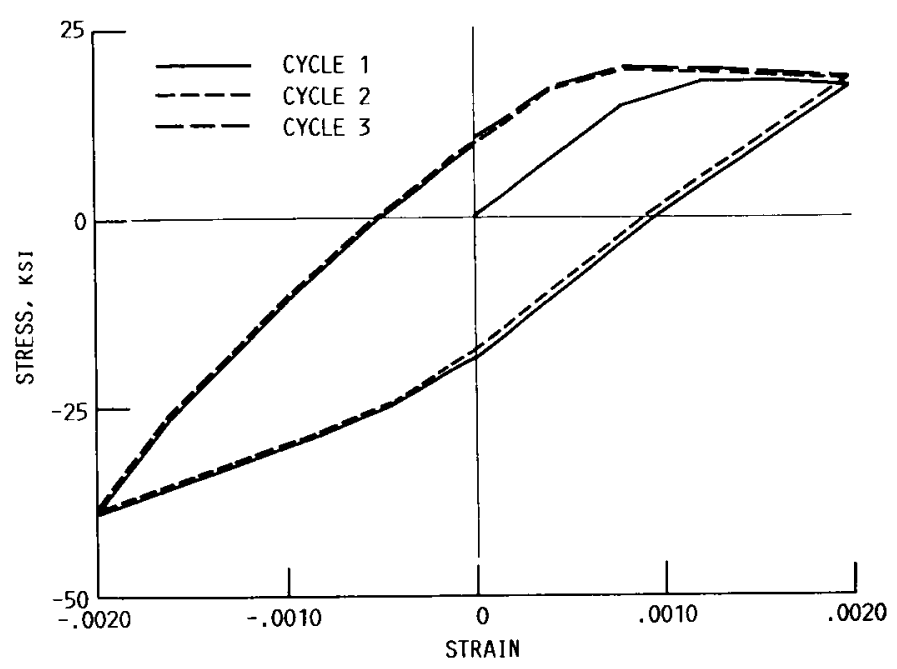

FIGURE 7. - HYSTERESIS LOOP USING ROBINSON'S MODEL-NONISOTHERMAL CASE (STRAIN RATE $=0.0004 / \mathrm{MIN}$ ), IN-PHASE THERMAL AND MECHANICAL LOADINGS.

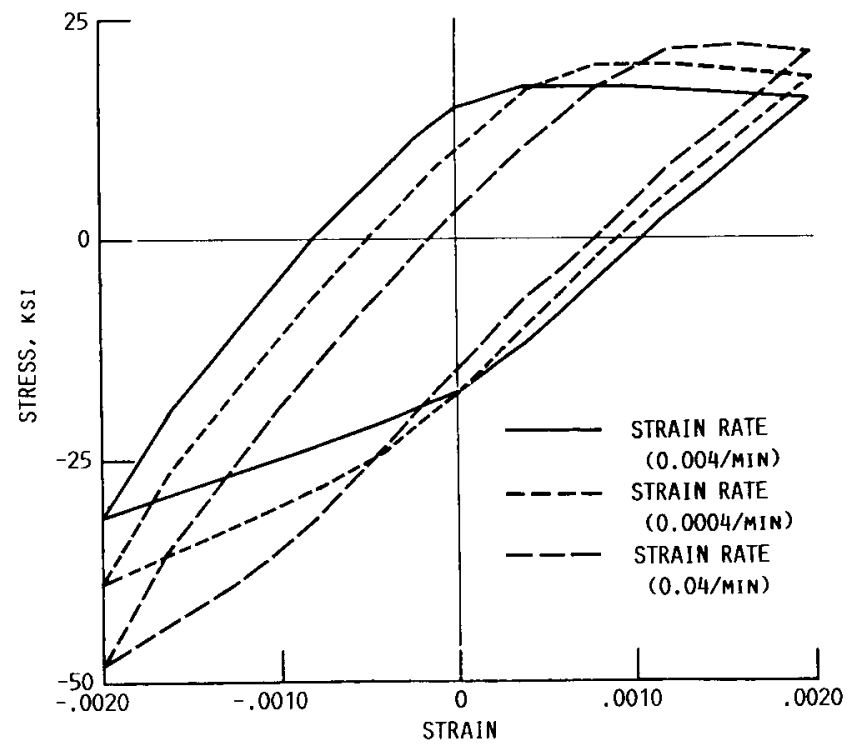

FIGURE 9. - HYSTERESIS LOOPS AT DIFFERENT STRAIN RATES USING ROBINSON'S MODEL-NONISOTHERMAL CASE. IN-PHASE THERMAL AND MECHANICAL LOADINGS.

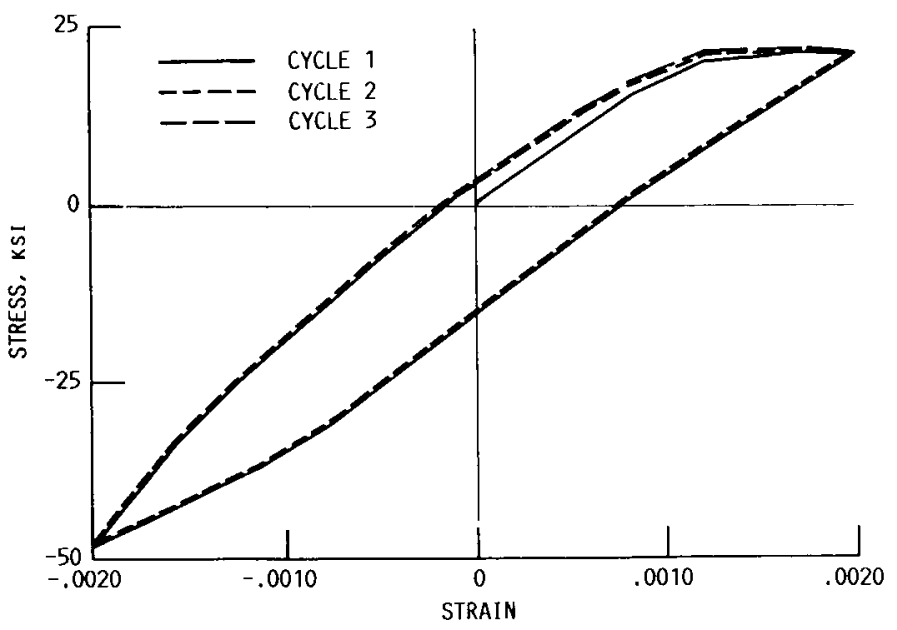

FIGURE 8. - HYSTERESIS LOOP USING ROBINSON'S MODEL-NONISOTHERMAL CASE (STRAIN RATE $=0.04 / \mathrm{MIN}$ ), IN-PHASE THERMAL AND MECHANICAL LOADINGS.

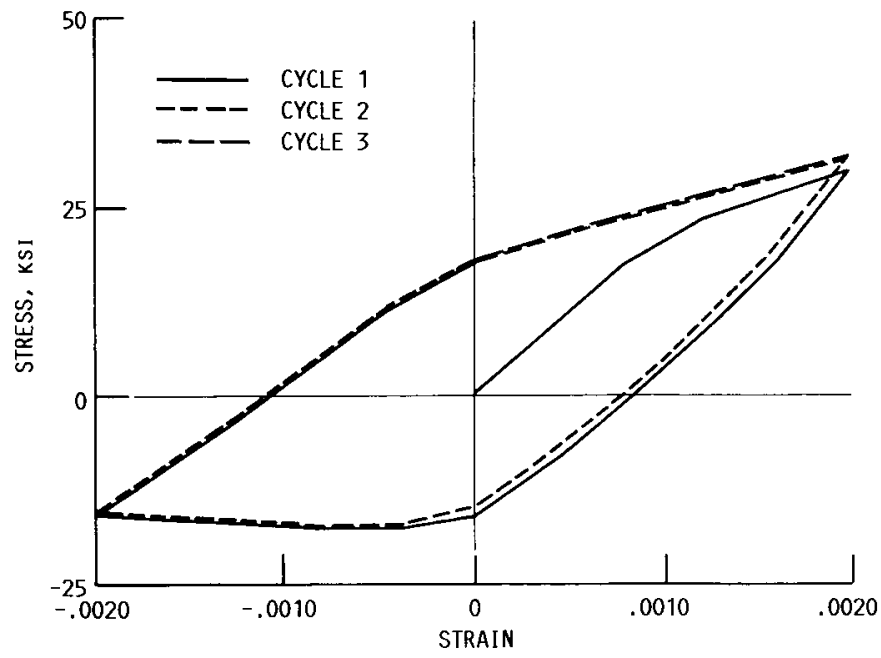

FIGURE 10. - HYSTERESIS LOOP USING ROBINSON'S MODEL-NONISOTHERMAL CASE (STRAIN RATE $=0.0004 / \mathrm{MIN}$ ), OUT-PHASE THERMAL AND MECHANICAL LOADINGS. 


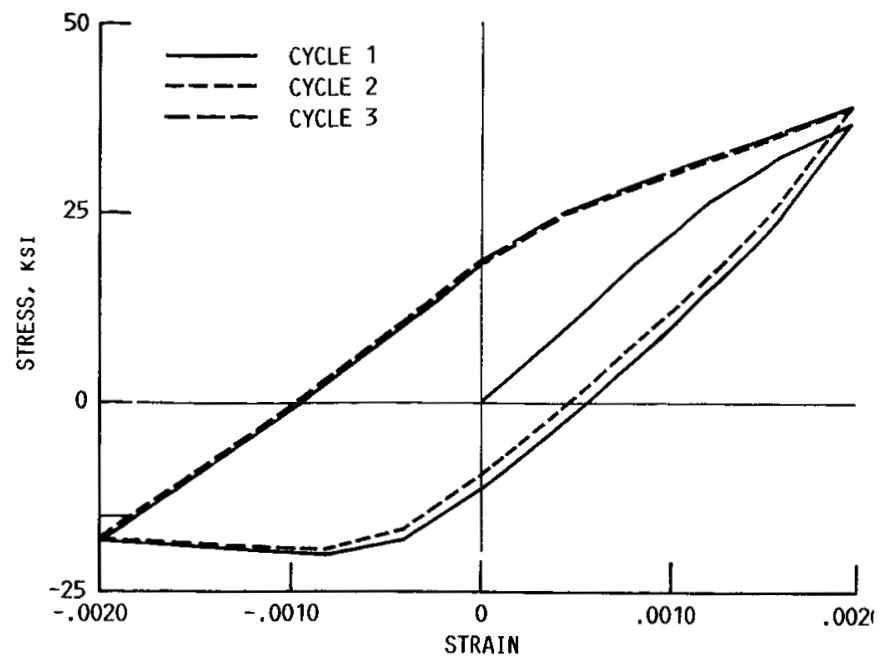

FIGURE 11. - HYSTERESIS LOOP USING ROBINSON'S MODEL-NONISOTHERMAL CASE (STRAIN RATE $=0.04 / \mathrm{MIN}$ ), OUT-PHASE THERMAL AND MECHANICAL LOADINGS.

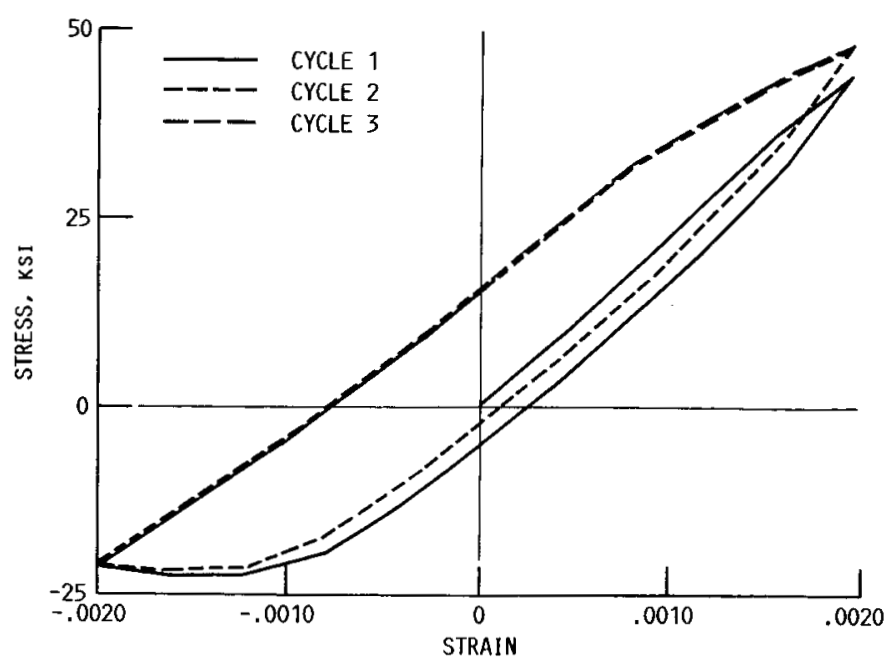

FIGURE 12. - HYSTERESIS LOOP USING ROBINSON'S MODEL-NONISOTHERMAL CASE (STRAIN RATE $=0.04 / \mathrm{MIN}$ ), OUT-PHASE THERMAL AND MECHANICAL LOADINGS.

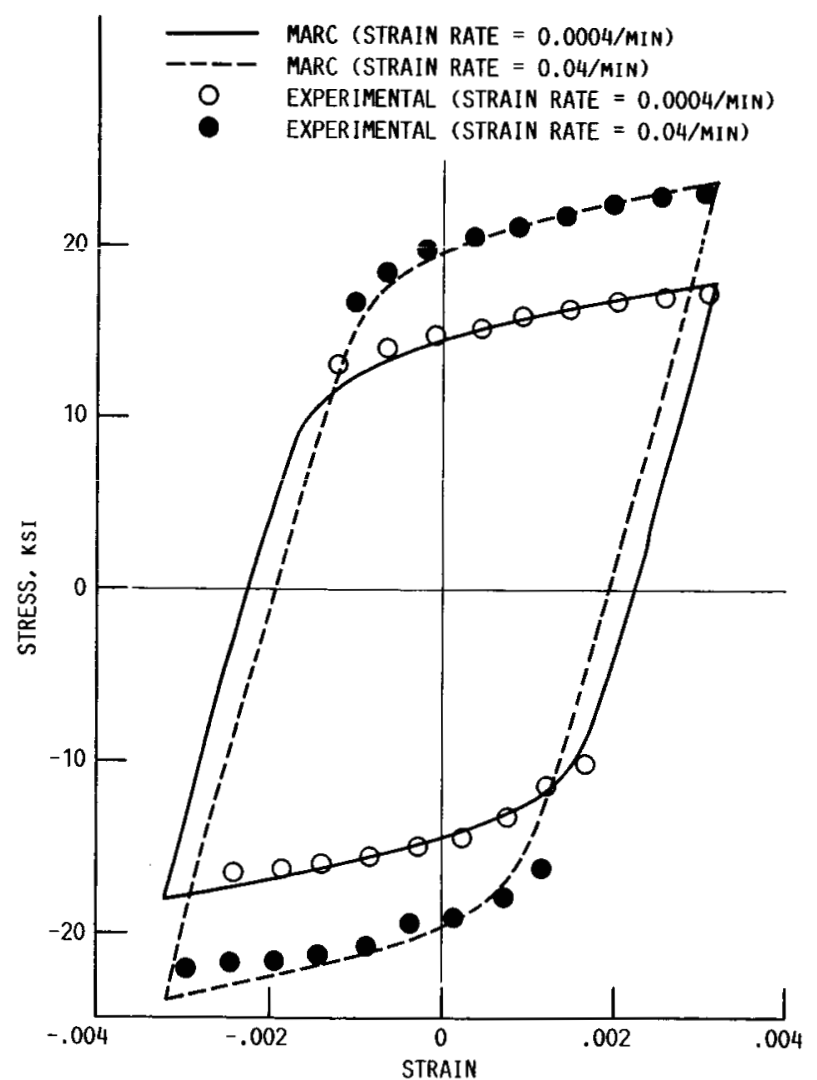

FIGURE 14. - COMPARISON OF MARC AND EXPERIMENTAL HYSTERESIS LOOPS $\left(1000^{\circ} \mathrm{F}\right)$. 


\section{OF POOR QUALTTY}

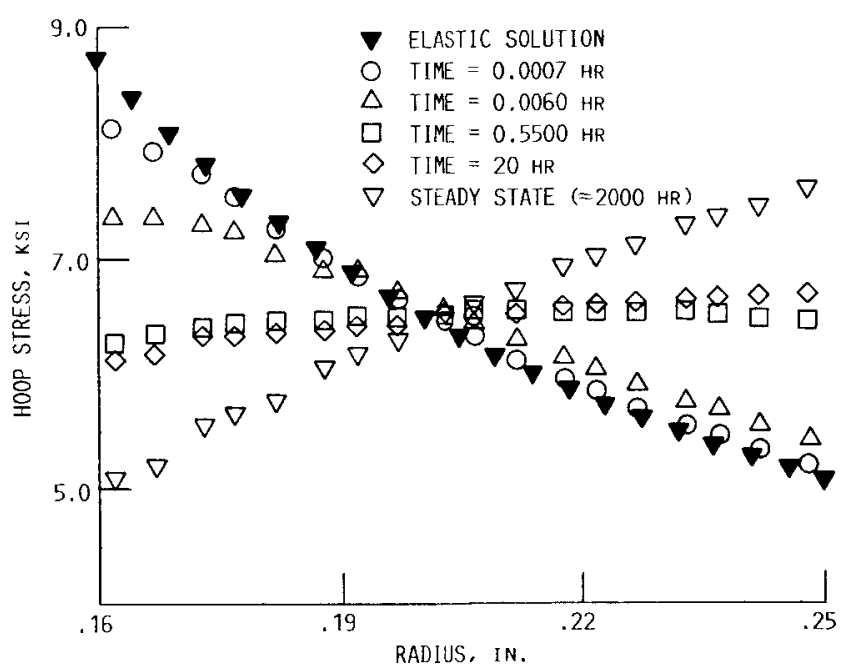

FISIIRE 15. - STRESS DISTRIBUTION IN AN INTERNALLY PRESSURIZED THICK CYLINDER USING ROBINSON'S MODEL (FEM-MARC SOLUTION).

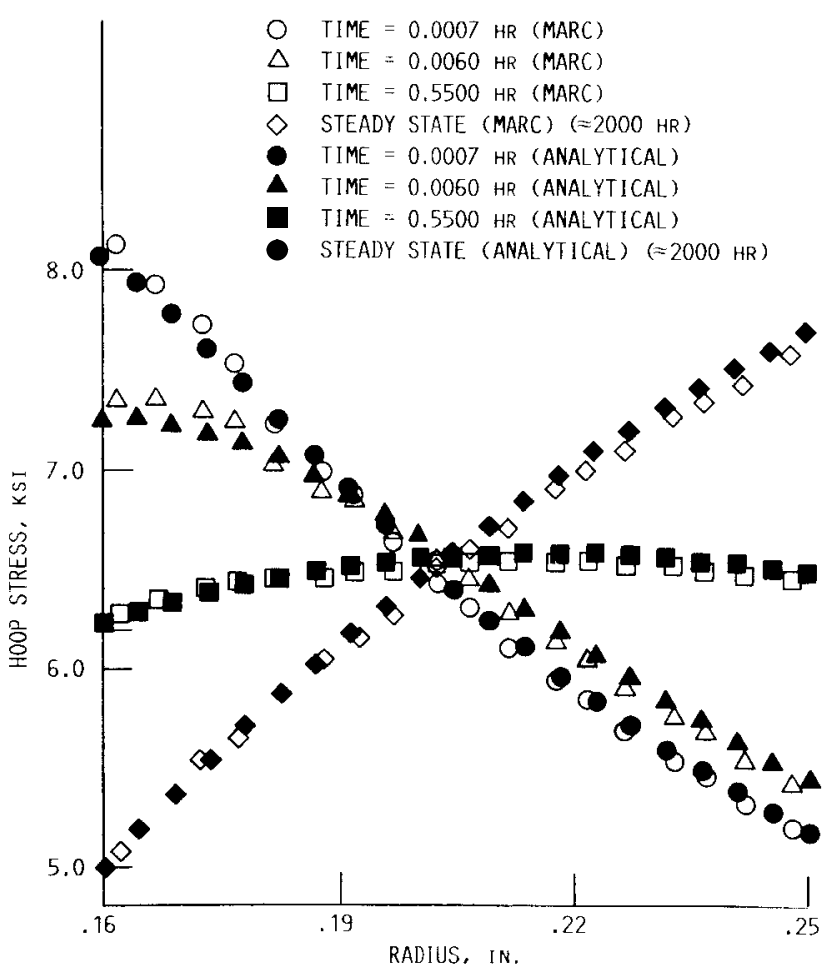

FIGURE 17. - COMPARISON OF MARC AND ANALYTICAL RESULTS FOR STRESS DISTRIBUTION IN AN INTERNALLY PRESSURILED THICK CYLINDER USING ROBINSON'S MODEL.

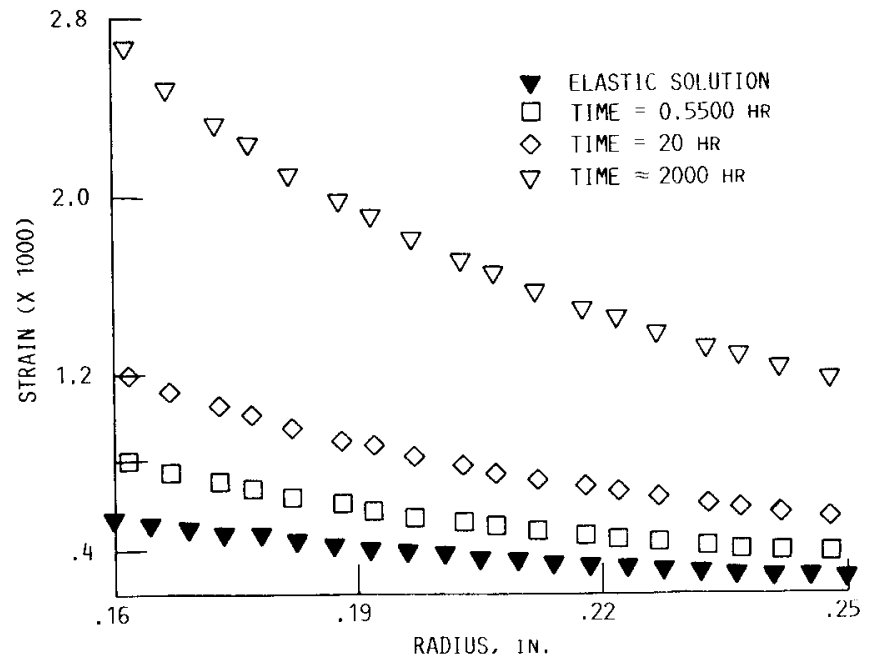

FIGURE 16. - STRAIN DISTRIBUTION IN AN INTERNALLY PRESSURIZED THICK CYLINDER USING ROBINSON'S MODEL (FEM-MARC SOLUTION).

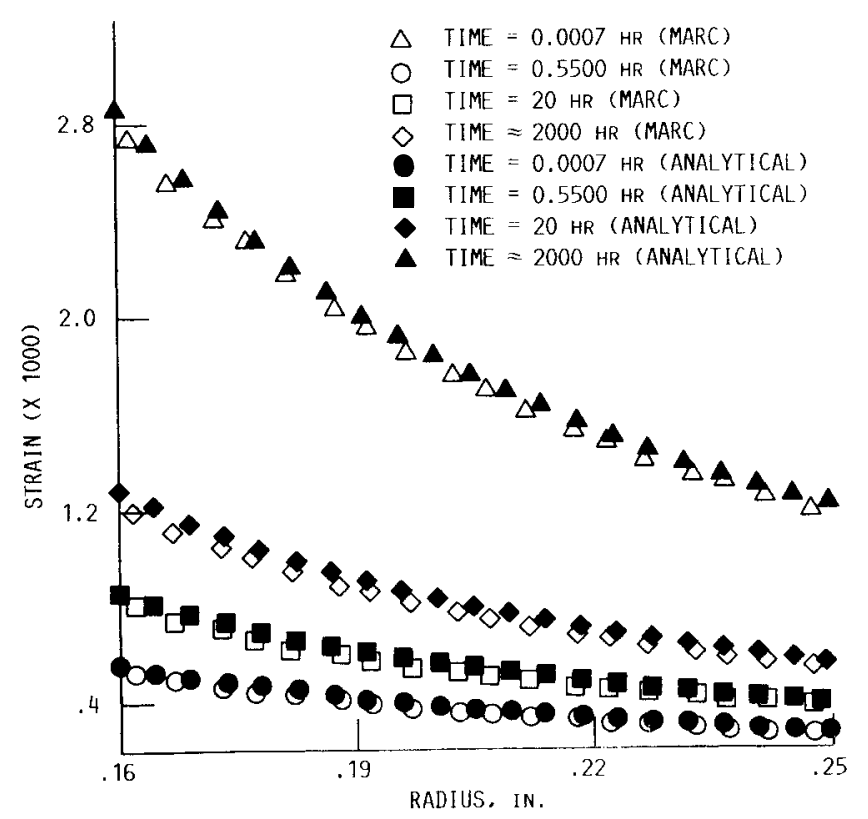

FIGURE 18. - COMPARISON OF MARC AND ANALYTICAL RESUI TS FOR STRAIN DISTRIBUTION IN AN INTERNALLY PRESSURIZED THICK CYLINDER USING ROBINSON'S MODEL. 


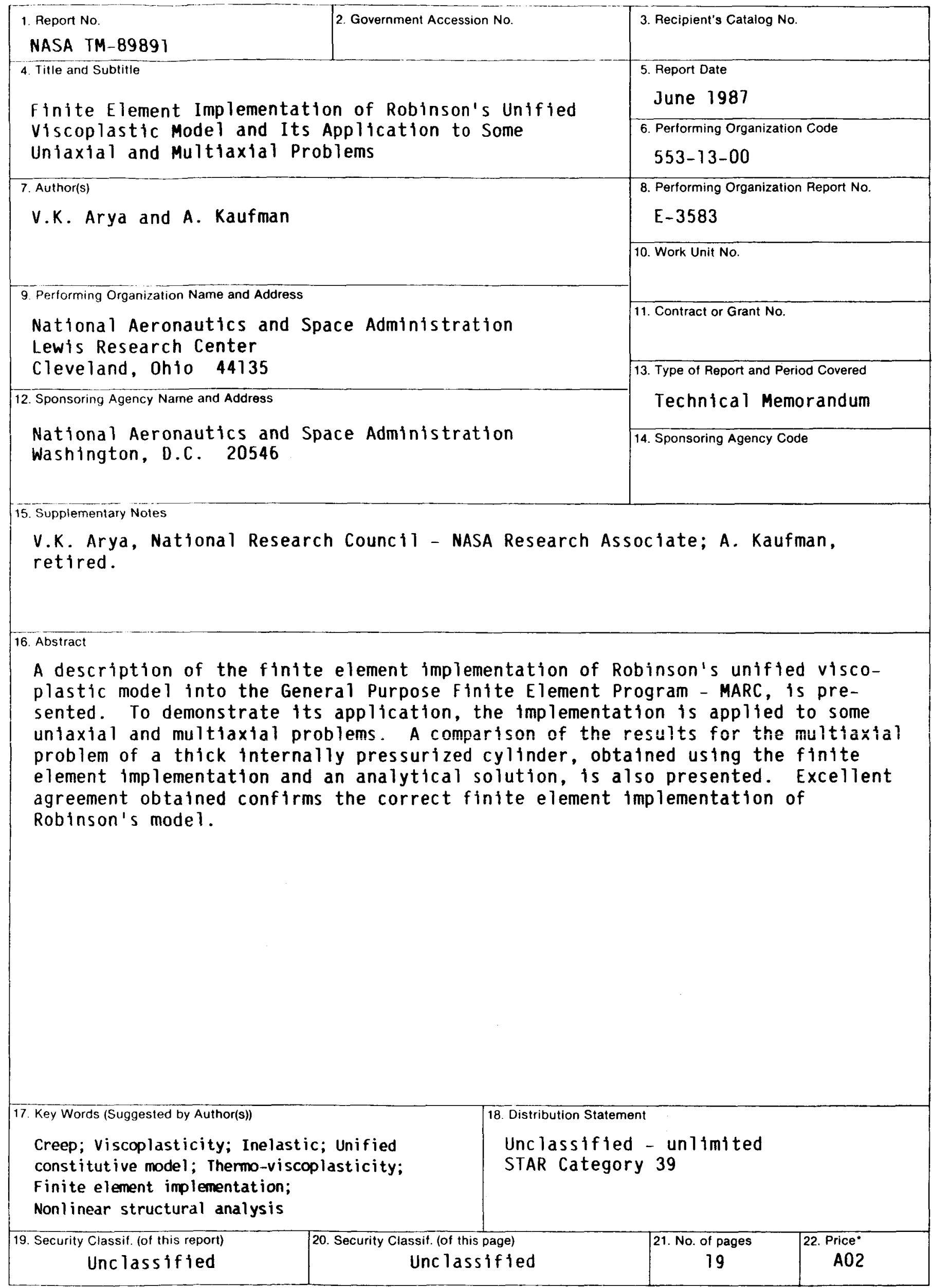

*For sale by the National Technical Information Service, Springfield, Virginia 22161 